\title{
RIN transfer in random distributed feedback fiber lasers
}

\author{
J. Nuño, ${ }^{*}$ M. Alcon-Camas, and J.D. Ania-Castañón \\ Instituto de Óptica Daza de Valdés, CSIC, Madrid, 28006, Spain \\ *javier.nuno@io.cfmac.csic.es
}

\begin{abstract}
We numerically investigate relative intensity-noise transfer from a noisy pump to the generated Stokes component in random distributed feedback ultralong Raman fiber lasers. Results show transfer levels comparable to those in distributed Raman amplification and cavity-based ultralong Raman fiber lasers, but with some unique spectral features.
\end{abstract}

(C) 2012 Optical Society of America

OCIS codes: (060.3510) Lasers, fiber; (060.4370) Nonlinear optics, fibers; (190.5650) Raman effect.

\section{References and links}

1. S. K. Turitsyn, S. A. Babin, A. E. El-Taher, P. Harper, D. V. Churkin, S. I. Kablukov, J. D. Ania-Castañón, V. Karalekas, and E. V. Podivilov, "Random distributed feedback fibre laser," Nat. Photonics 4, 231-235 (2010), http://www.nature.com/nphoton/journal/v4/n4/abs/nphoton.2010.4.html.

2. J. D. Ania-Castañón, T. J. Ellingham, R. Ibbotson, X. Chen, L. Zhang, and S. K. Turitsyn, "Ultralong Raman fiber lasers as virtually lossless optical Media," Phys. Rev. Lett. 96, 023902 (2006), http://prl.aps.org/abstract/PRL/v96/i2/e023902.

3. A. E. El-Taher, P. Harper, S. A. Babin, D. V. Churkin, E. V. Podivilov, J. D. Ania-Castañón, and S. K. Turitsyn, "Effect of Rayleigh-scattering distributed feedback on multiwavelength Raman fiber laser generation," Opt. Lett. 36, 130-132 (2011), http://www.opticsinfobase.org/ol/abstract.cfm?URI=ol-36-2-130.

4. A. E. El-Taher, M. Alcon-Camas, S. A. Babin, P. Harper, J. D. Ania-Castañón, and S. K. Turitsyn, "Dualwavelength, ultralong Raman laser with Rayleigh-scattering feedback," Opt. Lett. 35(7), 1100-1102 (2010), http://www.opticsinfobase.org/ol/abstract.cfm?uri=ol-35-7-1100.

5. H. Martins, M. B. Marques, and O. Frazão, "300 km-ultralong Raman fiber lasers using a distributed mirror for sensing applications," Phys. Rev. Lett. 19, 18149-18154 (2011), http://www.opticsinfobase.org/oe/fulltext.cfm?uri=oe-19-19-18149.

6. C. R. S. Fludger, V. Handerek, and R. J. Mears, "Pump to signal RIN transfer in Raman fiber amplifiers," J. Lightwave Technol. 19, 8, 1140-1148 (2001), http://ieeexplore.ieee.org/xpl/articleDetails.jsp?arnumber=939794.

7. B. Bristiel, S. Jiang, P. Gallion, and E. Pincemin, "New model of noise figure and RIN transfer in fiber Raman amplifiers," IEEE Photon. Technol. Lett. 18(8), 980-982 (2006), http://ieeexplore.ieee.org/xpl/articleDetails.jsp?arnumber=1613990.

8. M. Alcon-Camas and J. D. Ania-Castañón, "RIN transfer in 2nd-order distributed amplification with ultralong fiber lasers," Opt. Express 18(23), 23569-23575 (2010), http://www.opticsinfobase.org/oe/abstract.cfm?uri=oe-18-23-23569.

9. M. Krause, S. Cierullies, H. Renner, and E. Brinkmeyer, "Pump-to-Stokes RIN transfer in Raman fiber lasers and its impact on the performance of co-pumped Raman amplifiers," Opt. Commun. 260(2), 656-661 (2006), http://dx.doi.org/10.1016/j.optcom.2005.10.077.

10. D. V. Churkin, S. A. Babin, A. E. El-Taher, P. Harper, S. I. Kablukov, V. Karalekas, J. D. Ania-Castañń, E. V. Podivilov and S. K. Turitsyn, "Raman fiber lasers with a random distributed feedback based on Rayleigh scattering," Phys. Rev. A 82(3), 033828 (2010), http://pra.aps.org/abstract/PRA/v82/i3/e033828.

11. V. Karalekas, J. D. Ania-Castañń, P. Harper, S. A. Babin, E. V. Podivilov, and S. K. Turitsyn, "Impact of nonlinear spectral broadening in ultra-long Raman fibre lasers," Opt. Express 15, 16690-16695 (2007), http://www.opticsinfobase.org/oe/abstract.cfm?URI=oe-15-25-16690.

\#175553 - \$15.00 USD Received 5 Sep 2012; revised 11 Oct 2012; accepted 11 Oct 2012; published 19 Nov 2012

(C) 2012 OSA

19 November 2012 / Vol. 20, No. 24 / OPTICS EXPRESS 27376 


\section{Introduction}

Random distributed feedback ultralong Raman fiber lasers (RDF-RFL) relying on distributed Rayleigh backscattering [1] have become a very attractive concept over the last couple of years thanks to their unique properties, combining output characteristics similar to those of ultralong cavity fiber lasers [2] with others such as a wide tuneability and multi-wavelength operation [3]. Potential practical applications range from communications to distributed optical fiber sensing (DOFS) [4, 5], where they have proven useful for remote and non-contact sensing providing information of large critical infrastructures in very harsh environments.

Relative intensity noise (RIN), describes the instability in the power level of a optical signal. In systems based on Raman amplification, the RIN of the pump lasers can be transferred to the signal. Due to its fast gain dynamics, this transference is a potentially important source of impairment of these amplification systems [6].

In this paper, we will provide an accurate analysis of the RIN transfer in a RDF-RFLs, including the evolution of the RIN transfer function along the fiber and its dependence on the length and the pump power. Moreover this performance is compared with that of other Raman lasers, as the ultralong cavities, operating in similar conditions.

\section{Results and discussion}

The study of the evolution of RIN transfer in Raman amplifiers has been undertaken in several occasions $[6,7]$. Following the example set in [8] it is possible to define a complete set of ordinary differential equations (ODEs) that defines the evolution of the average powers and the intensity noise components for the pumps and the signals, including the effects of depletion, attenuation, multiple Rayleigh backscattering and the amplified spontaneous emission (ASE). This set of equations is:

$$
\begin{gathered}
\frac{d P_{p}^{ \pm}}{d z}=\mp \alpha_{p} P_{p}^{ \pm} \mp \frac{v_{p}}{v_{s}} g\left(P_{s}^{+}+P_{s}^{-}+4 h v_{s} \Delta v_{s}\left(1+\frac{1}{e^{h\left(v_{p}-v_{s}\right) / K_{B} T}-1}\right)\right) P_{p}^{ \pm} \pm \varepsilon_{p} P_{p}^{\mp} \\
\frac{d P_{s}^{ \pm}}{d z}=\mp \alpha_{s} P_{s}^{ \pm} \pm g\left(P_{s}^{ \pm}+2 h v_{s} \Delta v_{s}\left(1+\frac{1}{e^{h\left(v_{p}-v_{s}\right) / K_{B} T}-1}\right)\right)\left(P_{p}^{+}+P_{p}^{-}\right) \pm \varepsilon_{s} n_{s}^{\mp} \\
\frac{d n_{p}^{ \pm}}{d z}+i d_{p}^{ \pm} \omega n_{p}^{ \pm}=\mp \alpha_{p} n_{p}^{ \pm} \mp \frac{v_{p}}{v_{s}} g\left(n_{s}^{+}+n_{s}^{-}\right) P_{p}^{ \pm} \mp \frac{v_{p}}{v_{s}} g\left(P_{s}^{+}+P_{s}^{-}\right) n_{p}^{ \pm} \pm \varepsilon_{p} n_{p}^{\mp} \\
\frac{d n_{s}^{ \pm}}{d z}+i d_{s}^{ \pm} \omega n_{s}^{ \pm}=\mp \alpha_{s} n_{s}^{ \pm} \pm g\left(n_{p}^{+}+n_{p}^{-}\right) P_{s}^{ \pm} \pm g\left(P_{p}^{+}+P_{p}^{-}\right) n_{s}^{ \pm} \pm \varepsilon_{s} n_{s}^{\mp}
\end{gathered}
$$

where subscripts $\mathrm{p}$ and $\mathrm{s}$ are referring to the pump and signal respectively and the signs + and - correspond to components propagating in the forward and backward directions. $P_{i}$ are the averages powers and $n_{i}$ the spectral density of the intensity noise. $\alpha_{i}$ are the attenuations, $g$ is the Raman gain and $\varepsilon_{i}$ are the Rayleigh coefficient. The optical frequency for each spectral component is represented by $v_{i}$ and the effective bandwidths by $\Delta v_{i} . K_{B}$ is Boltzmann's constant,h is Plank's constant and T is the absolute temperature of the fiber. Finally, $d_{i}^{ \pm}$take into account the different velocities in the transmission and they are defined as $d_{i}^{ \pm}=1 / v_{i} \mp 1 / v_{s}$ where $v_{i}$ are the group velocities for each wavelength. In Table 1, the values of the main parameters of the fiber are summarized. The RIN transfer function itself can be defined as:

\#175553 - \$15.00 USD Received 5 Sep 2012; revised 11 Oct 2012; accepted 11 Oct 2012; published 19 Nov 2012

(C) 2012 OSA

19 November 2012 / Vol. 20, No. 24 / OPTICS EXPRESS 27377 


$$
H_{R I N}(x)=\left|\frac{n_{s}(x)}{N_{\text {in }}}\right|
$$

where $N_{\text {in }}$ is the initial intensity noise of the pump laser. Here, we solve this model for the case of the symmetric, centrally-pumped RDF-RFL, first presented in [1] and whose scheme is depicted in Fig. 1. It consists of a span of standard fiber that is centrally pumped at 1455 $\mathrm{nm}$ to generate lasing at $1550 \mathrm{~nm}$. Thanks to the symmetry of this scheme, the problem can be simplified to solve the set of ODEs for only one arm of the laser (between the central point and one of the ends) considering as boundary conditions in the central point that the counterpropagating Stokes signal is reflected into the co-propagating one, in other words:

$$
\begin{gathered}
P_{p}^{+}(0)=P_{\text {in }}+\eta \times P_{p}^{-}(0) ; P_{s}^{+}(0)=\eta \times P_{s}^{-}(0) ; \\
n_{p}^{+}(0)=N_{\text {in }}+\eta \times n_{p}^{-}(0) ; n_{s}^{+}(0)=\eta \times n_{s}^{-}(0) .
\end{gathered}
$$

where $P_{i n}$ is the initial pump power, $N_{i n}$ its initial intensity noise and $\eta$ is the efficiency of the WDM-coupler in the middle point.

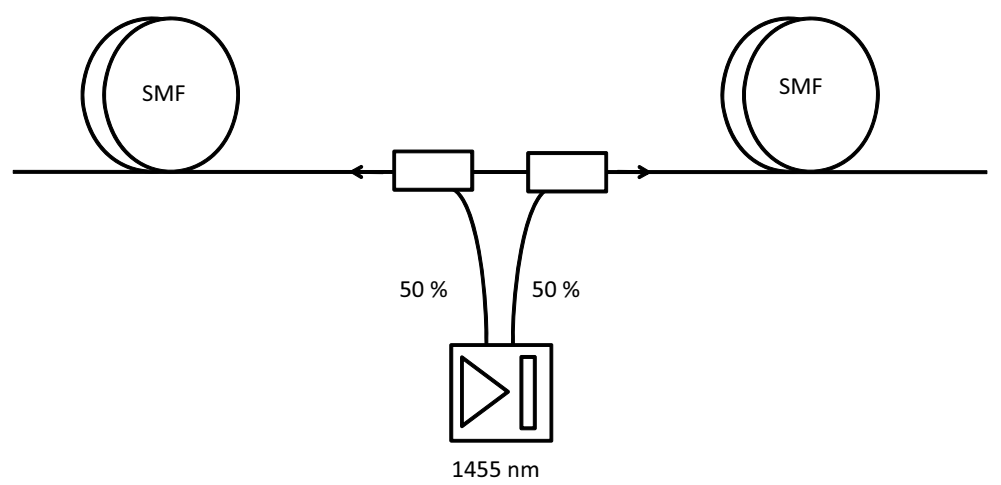

Fig. 1. Schematic for a centrally-pumped RDF-RFL.

Table 1. Main parameters in the simulation.

\begin{tabular}{|c|c|c|c|}
\hline wavelength $(\mathrm{nm})$ & attenuation $(\mathrm{dB} / \mathrm{Km})$ & Rayleigh coefficient $\left(\mathrm{m}^{-1}\right)$ & refraction index \\
\hline 1455 & 0.263 & $6.0 \times 10^{-8}$ & 1.4996 \\
1550 & 0.197 & $4.28 \times 10^{-8}$ & 1.5004 \\
\hline
\end{tabular}

Our analysis begins with the study of the evolution of the RIN noise transfer function across the gain medium, for a Stokes signal propagating from the left to the right of the laser. It is not usual to present this feature in the analysis of RIN performance in lasers, however it becomes relevant in ultralong structures, particularly if they are going to be used for distributed sensing, since the sensitivity and dynamic range will depend on the noise distribution along the line. Given that the pumping beam is launched bi-directionally from a central point, the results show two well-differentiated sections from the standpoint of a Stokes component propagating from left to right. In the first section, the Stokes signal is counter-propagating with the launched pump, with a small amount of co-propagating pump power being present due to Rayleigh backscattering. In the second section, past the mid-point, the Stokes signal is co-propagating with

\#175553 - \$15.00 USD Received 5 Sep 2012; revised 11 Oct 2012; accepted 11 Oct 2012; published 19 Nov 2012

(C) 2012 OSA

19 November 2012 / Vol. 20, No. 24 / OPTICS EXPRESS 27378 

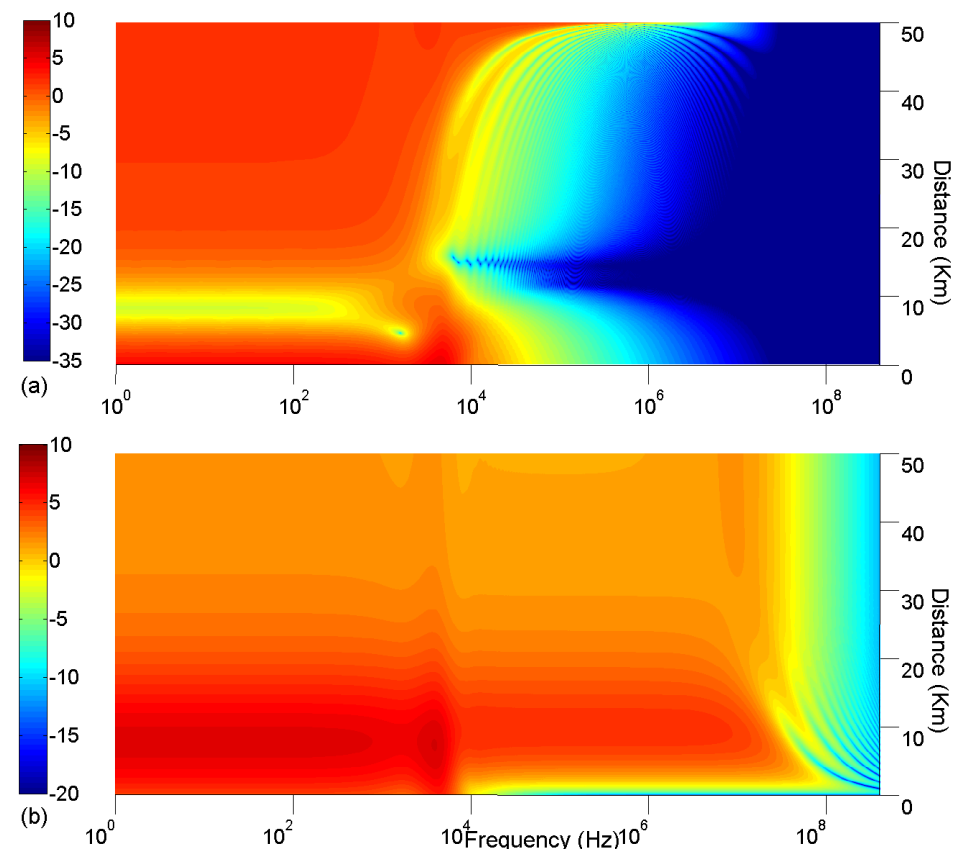

Fig. 2. Evolution of the RIN transfer versus frequency along the fiber length for the situation where the amplification is mainly counter-propagated (top) or co-propagated (bottom). The total pump power is $3 \mathrm{~W}$ and the total length is $100 \mathrm{Km}$.

the launched pump, although a small amount of Rayleigh-backscattered pump-power is presented. Both cases are depicted in Fig. 2. As it was expected, the cutoff frequency for the counter-propagating case is $10 \mathrm{KHz}$ while it is higher for the co-propagating case (approximately $80 \mathrm{MHz}$ ). On the other hand, the transfer figure remains almost uniform along the distance and, consequently, a sensitivity error produced by the RIN a will be similar in each point of the laser.

There is another special feature of this RIN transfer function, namely the appearance of small fluctuations in the spectrum after the value of the cutoff frequency for the counter-propagating case. Such fluctuations have been also observed on traditional Raman lasers [9] and they are caused by the interaction between the generated counter-propagated signals. Its separation (the frequency difference between oscillations) is equivalent to the resonant cavity frequency in traditional lasers, as it has been observed in the literature, whereas in the random laser it depends on the distance between the pump point and the observation point as: $f_{c}=1 /(2 \pi L)$ where $\mathrm{L}$ is this distance. These oscillations have also much smaller amplitude in the case of RDF lasers, and are often non-observable in standard-fibre configurations, except for cavities longer than $80 \mathrm{~km}$ and pump powers above $2.8 \mathrm{~W}$.

Secondly, the most important parameters of our system have been varied with the objective of finding the optimal configurations that could minimize noise transfer while still offering a meaningful performance in terms of output power. In Fig. 3 total pump power and total length have been varied between 2 and 3.8W and between 30 and $200 \mathrm{Km}$, respectively. Note that the higher the pump power launched, the less RIN is transferred. Nevertheless, increasing the pump power beyond $3.8 \mathrm{~W}$ is not practical, since that is the threshold for the second Raman Stokes, which would cause a rapid depletion of the signal. Moreover, the cut-off frequency is slightly, but linearly, dependent on the pump power. Finally, the most important source of 

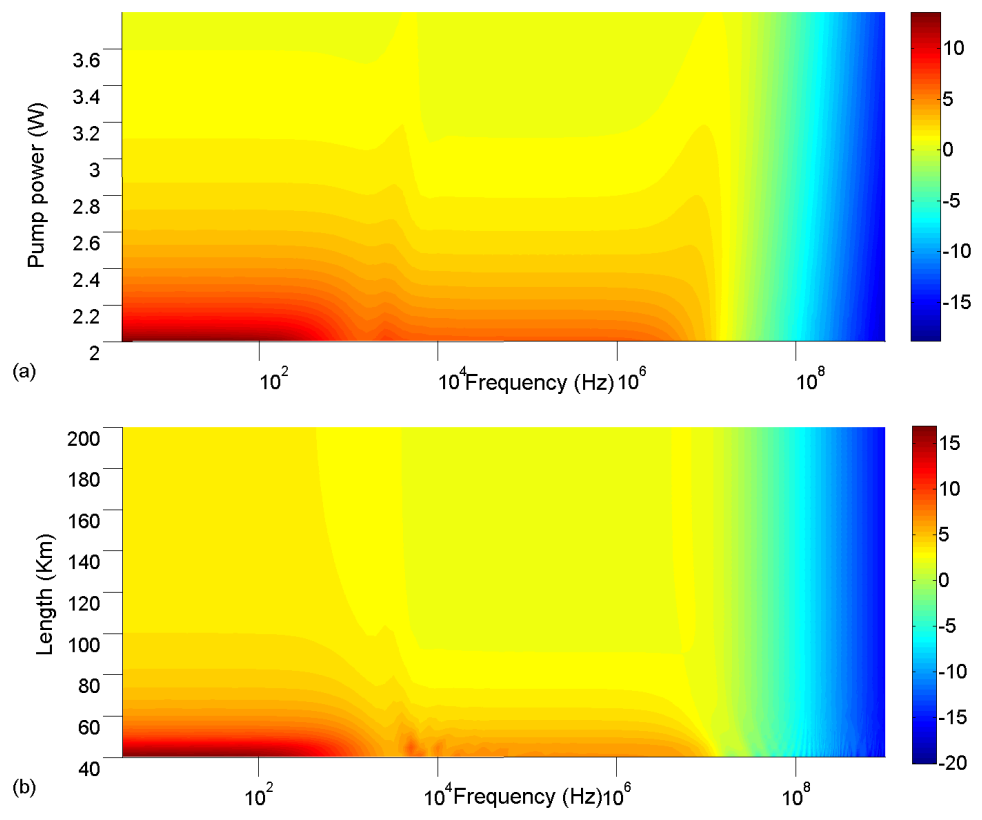

Fig. 3. Variation of the RIN transfer vs. frequency with the total pump power (top) and the total length (bottom). In the first case, the total length is $100 \mathrm{Km}$ and, in the second case, the total pump power is $3 \mathrm{~W}$.

impairment to be considered when the pump power increases is related with the fluctuation described previously: for pump powers higher than $2.8 \mathrm{~W}$ the peak of maximum RIN transfer is reached in the oscillations themselves (usually in one immediately after the drop corresponding to the cut-off frequency for the counter-propagating component).

As it can be seen in Fig. 3, minimum RIN transfer is obtained for the longest laser. However, from our pump and signal power calculations we can observe that the peak output power is reached at $58 \mathrm{Km}$ length. The cut-off frequency itself is not affected by the fiber length. Regarding the oscillations, their separation decrease with length, and so do their peak intensities.

Although this configuration is mirrorless, and so no reflector characteristics can be optimized, it is interesting to know if some control over RIN transfer can be achieved by using fibers with different Rayleigh scattering parameters. In this case, the maximum RIN transfer is inversely proportional to Rayleigh scattering, as is output power. Generally speaking, the RIN transfer function is in most cases below $10 \mathrm{~dB}$, comparable to those seen in distributed Raman amplification systems based on standard fiber operating at similar gain values and power levels.

Finally, a comparison between RDF-RFL and an ultra-long Raman laser pumped from both ends is provided. In both configurations, we consider the transfer between the pump at $1455 \mathrm{~nm}$ and the generated signal at $1550 \mathrm{~nm}$ along a $100 \mathrm{~km}$ fiber span. In order to obtain an equivalent power level for the generated Stokes signal in both lasers, the pump power in the shown example needs to be higher in the RDF-RFL laser $(2.7 \mathrm{~W})$ than in the ultra-long cavity-based RFL (1.7 W). In both cases, we suppose an identical initial RIN level for the pumps. The results are shown in Fig. 4. The average level of the RIN transfer is similar in both configurations but the oscillations are noticeable only in the ultra-long cavity laser case. For this reason, the use of the ultra-long cavity configuration can be useful to reduce RIN transfer of specific spectral components in the pump laser, as we can chose the length of the cavity to minimize its effect at 
a particular location. Obviously, ultralong cavities also have a clear advantage in terms of conversion efficiency except in the case of extreme cavity lengths [1, 10]. In cases these two issues are not a priority, the use of an RDF-RFL configuration could prove more convenient given the reduced level of the RIN oscillations, their lack of a power-dependent effective reflector response [11], and their comparative advantage in terms of tuneability.
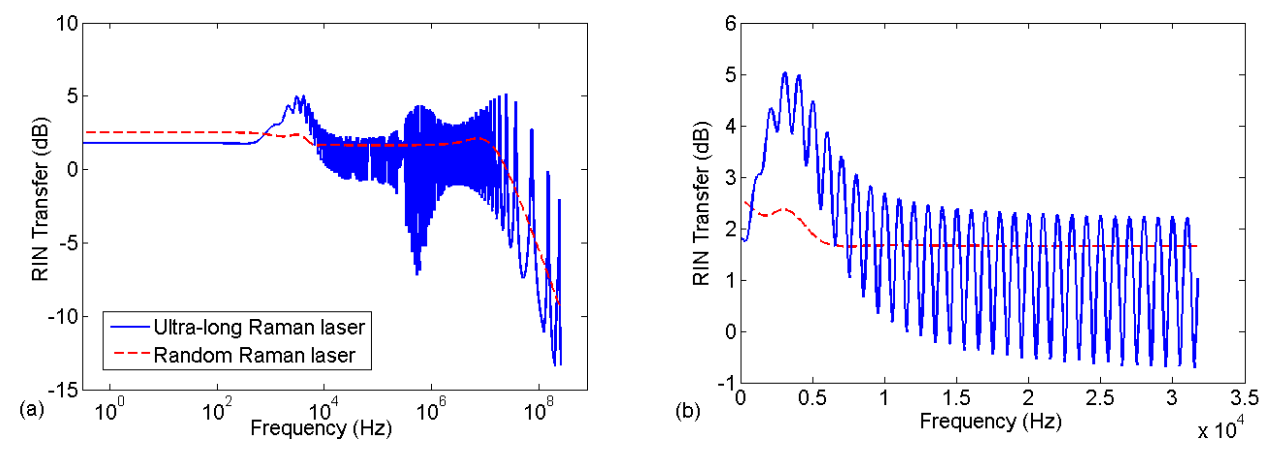

Fig. 4. Comparative between the RIN transfer for the RDF-RFL configuration and the traditional RFL in a logarithmic scale (left side) and a linear scale (right side).

\section{Conclusion}

The RIN transfer function for a one-level RDF fiber laser has been investigated. Cut-off frequencies for the co- and counter-propagating components appear as expected, at positions independent of the fiber length and showing a weak linear dependence with the pump power. A limited control over RIN transfer function of the laser can be applied at the design stage through appropriate selection of fibers and choice of length. Our results show that optimal configurations to minimize noise transfer imply the use of higher pump power and long fibers, but not long enough to allow for the quick generation of additional Stokes, which would cause a rapid depletion of the signal. For cavities longer than $80 \mathrm{~km}$ and pump powers above $2.8 \mathrm{~W}$, fluctuations in the RIN transfer appear, which seems to be related to the interaction of the copropagating signal with the counter-propagating seed generated on the symmetric arm. These fluctuations are, nevertheless, generally smaller in amplitude than those appearing in cavitybased laser configurations. Overall, the behavior of RIN transfer in RDF-RFL should not impede their use in communication schemes and distributed optical fiber sensing applications, but as in the case of traditional (cavity) ultra-long Raman fiber lasers, configurations that minimize RIN transfer should be chosen over noisier ones.

\section{Acknowledgments}

The authors wish to thank the financial support of Ministerio de Economía y competividad (MINECO) through grant TEC2011-27314. The work of Javier Nuño is partly supported by the Ministerio de Economía y competividad (MINECO) through an FPI Fellowship. 